\title{
DIAGNÓSTICOS, INTERVENÇÕES E RESULTADOS DE ENFERMAGEM PARA SUBSIDIAR A CONSULTA DE ENFERMAGEMÀ CRIANÇA
}

Ingrid Pujol Hanzen ${ }^{1}$, Silvana dos Santos Zanotelli', Elisangela Argenta Zanatta ${ }^{1}$

Objetiuo: apresentar diagnósticos (DE), intervenções (IE) e resultados de enfermagem (RE) construídos para subsidiar a Consulta de Enfermagem à criança de zero a dois anos de idade na Atenção Primária à Saúde com base na Classificação Internacional para a Prática de Enfermagem (CIPE®) e na teoria de Wanda de Aguiar Horta. Método: pesquisa qualitativa, do tipo pesquisa-ação, realizada com sete enfermeiras que atuam na APS de um município do oeste catarinense. Os DE, IE e RE foram validados pelo Índice de Validade de Conteúdo. Resultados: Foram criados e validados 19 enunciados de DE, com intervenções e resultados esperados com base na CIPE®, voltados para crianças de zero a dois anos de idade.

Conclusão: A criação de DE, IE e RE respalda e qualifica o trabalho do enfermeiro, empoderando-o e dando cientificidade às suas ações.

Palauras-chave: Enfermeiro; Processo de Enfermagem; Diagnósticos de Enfermagem; Puericultura.

\section{DIAGNOSTICS, INTERVENTIONS AND NURSING RESULTS TO SUBSIDY THE NURSING CONSULTATION OF THE CHILD}

Objective: to present Nursing diagnosis (ND), interventions (NI) and results (NR) to assess the child from zero to two years old on Primary Health Care, based on the International Classification for Nursing Practice (ICNP). Method: qualitative research, of the action-research type, with seven nurses that act on PHC of a city in the west of Santa Catarina. ND, NI and NR were validated through Content Value Índex. Results: Nineteen ND statements were created and validated, with ICNP®-based interventions and expected outcomes targeting children from zero to two years old..

Conclusion: Creating ND, NI and NR supports and qualifies the work of nurses, empowering them and giving scientificity to their actions

Key-words: Nurse; Nursing Process; Nursing Diagnosis; Child Care.

\section{DIAGNÓSTICO, INTERVENCIONES Y RESULTADOS DE ENFERMERÍA PARA SUBVENCIONAR LA CONSULTA DE ENFERMERÍA} INFANTIL

Objetivo: presentar diagnósticos (DE), intervenciones (IE) y resultados (RE) de enfermería para subvencionar la atención de niños de cero a dos años en Atención Primaria de Salud (APS), según la Clasificación Internacional para la Práctica de Enfermería (CIPE®). Método: investigación cualitativa, del tipo de investigación de acción, realizada con siete enfermeras que trabajan en APS de un municipio del oeste de Santa Catarina. DE, IE y RE fueron validados por el Índice de Validez del Contenido. Resultados: Diecinueve declaraciones DE fueron creadas y validadas, con intervenciones basadas en CIPE® y resultados esperados dirigidos a niños de cero a dos años.

Conclusión: La creación de DE, IE y RE apoya y califica el trabajo de las enfermeras, les da poder y les da carácter científico a sus acciones.

Palabras llave: enfermera; Proceso de enfermería; Diagnósticos de enfermería; Cuidado de niños.

${ }^{1}$ Universidade do Estado de Santa Catarina- UDESC

Autor Correspondente: Ingrid Pujol Hanzen. Email: ingridhanzen@yahoo.com.br 


\section{INTRODUÇÃO}

A criança faz parte do grupo prioritário de atenção à saúde, o que pressupõe garantir a implementação de uma assistência sistematizada que favoreça o processo de cuidado. A Política Nacional de Atenção Básica (PNAB) define a Atenção Primária à Saúde (APS) como o nível de atenção à saúde que representa o primeiro contato com as crianças e famílias oferecendo serviços e ações que visam a atenção integral à criança com ações voltadas à promoção e reabilitação da saúde, prevenção e cura de agravos, garantindo assim a longitudinalidade do cuidado ${ }^{(1,2)}$.

Para consolidação da APS, tem-se a Estratégia Saúde da Família (ESF), articuladora das relações entre o indivíduo, família, comunidade e equipe de saúde. Na ESF, uma das estratégias utilizada para o acompanhamento da saúde da criança é o Programa de Puericultura, que contempla, de forma integral, a vigilância do crescimento, desenvolvimento e processos de saúde e doença ${ }^{(2,3)}$.

Na realização da Consulta de Enfermagem (CE) em puericultura, o enfermeiro, tem papel fundamental na criação de vínculos e na realização de atendimentos humanizados e qualificados, possuindo como ferramenta de trabalho a Sistematização da Assistência de Enfermagem (SAE) instituída pela resolução 358/2009 do Conselho Federal de Enfermagem (COFEN) que dispõe sobre a SAE e a implementação do Processo de Enfermagem $(\mathrm{PE})^{(4)}$. O PE, quando realizado em instituições prestadoras de serviços ambulatoriais de saúde, domicílios, escolas e associações comunitárias corresponde à CE.

Para que a CE se desenvolva de maneira sistematizada, precisa seguir as etapas do PE: Coleta de dados de Enfermagem (ou Histórico de Enfermagem), Diagnóstico de Enfermagem, Planejamento de Enfermagem, Implementação e avaliação de enfermagem ${ }^{(4)}$. Para subsidiar as etapas de DE, planejamento, implementação e avaliação é importante adotar um Sistema de Linguagem Padronizado (SLP) que ordena termos ou expressões que compõem os diagnósticos, intervenções, avaliações e resultados esperados - componentes do $\mathrm{PE}^{(5)}$.

Dentre os SLP destaca-se a Classificação Internacional para a Prática de Enfermagem (CIPE $®$ ), uma terminologia padronizada que representa o domínio da prática de enfermagem no âmbito mundial. É uma tecnologia de informação que proporciona a coleta, armazenamento, análise de dados de enfermagem em diversos cenários ${ }^{(6)}$.

Na prática diária é perceptível que a CE sistematizada, ainda é pouco usada nos processos de trabalho, sendo que o motivo vai desde o pouco conhecimento acerca do PE, insegurança para realizá-la até a falta de tempo. Com isso a elaboração de um instrumento que contemple DE, IE e RE otimiza e qualifica o trabalho do enfermeiro.

Esse manuscrito tem como objetivo apresentar DE, IE e RE construídos para subsidiar a CE à criança de zero a dois anos na APS, com base na CIPE® e na teoria de Wanda de Aguiar Horta. É parte da Dissertação apresentada ao Mestrado Profissional em Enfermagem na Atenção Primária à Saúde da Universidade do Estado de Santa Catarina e integra um macroprojeto de pesquisa intitulado "Estratégias para a implementação da Sistematização da Assistência de Enfermagem no cuidado à mulher e à criança", contemplado pelo Edital CAPES/COFEN no 27/2016.

\section{MÉTODO}

\section{Tipo de estudo}

Pesquisa-ação com abordagem qualitativa.

\section{Local do Estudo}

A pesquisa foi realizada em um município do oeste catarinense de junho a agosto de 2018.

\section{Participantes da Pesquisa}

Foram selecionadas sete enfermeiras a partir dos seguintes critérios de inclusão: atuar diretamente no cuidado à criança no município em estudo, estar cadastrada no Cadastro Nacional de Estabelecimentos de Saúde (CNES), possuir no mínimo seis meses de atuação como enfermeira. Foram excluidas as que estavam afastados por licença ou férias no momento da coleta dos dados.

\section{Coleta de dados}

Foram utilizadas seis etapas adaptadas de Thiollent ${ }^{(7)}$ : fase exploratória, diagnóstico de situação, coleta de dados, seminários integradores, planejamento de qualificação dos profissionais enfermeiros, publicização. Nesse manuscrito, será descrita a etapa dos seminários integradores.

Foram realizados quatro seminários integradores, conduzidos pela técnica de grupo focal $(\mathrm{GF})^{(8)} \mathrm{com}$ duração aproximada de três horas cada um.

\section{Procedimentos de interpretação e discussão dos dados}

O primeiro seminário foi construída a primeira etapa da CE (a anamnese e exame físico). No segundo, foi realizada a validação do primeiro e, na sequência, realizada a discussão e construção de um roteiro para o exame físico. Assim como no seminário anterior, o instrumento construído foi testado pelas enfermeiras por um período de 15 dias durante as consultas em puericulturas agendadas conforme rotina de cada Unidade Básica de Saúde (UBS). 
No terceiro seminário, foi validado e aprovado o roteiro do exame físico e, na sequência foi realizada uma capacitação de quatro horas sobre a CIPE®, modelo de sete eixos, versão 2017. Para essa capacitação partiu-se da elaboração de uma lista dos principais problemas evidenciados pelas enfermeiras no seu cotidiano de atendimento às crianças. Para cada problema elencado foram selecionados termos que integram o eixo Foco e o eixo Julgamento (minimamente), ou ainda representados por um Achado clínico. Após, foi realizada a construção dos DE, IE e RE fundamentados na CIPE $®$, ancorados na experiência e julgamento clínico das enfermeiras possibilitando a aproximação da teoria com a realidade e necessidades locais. Os DE, IE e RE foram testados pelo período de 30 dias no decorrer das consultas de puericultura de rotina das enfermeiras para verificar sua aplicabilidade na prática.

No quarto encontro, foi realizada a validação das etapas de Coleta de dados, DE, IE e RE por meio do Índice de Validade do Conteúdo (IVC). Para a validação foi construída uma escala likert com pontuação de um a quatro, sendo considerado aceitável o score igual ou superior a 0,80 de taxa de concordância ${ }^{(9)}$. Nesse trabalho o IVC foi de 0,80.

Todas as etapas construidas, testadas e validadas tiveram como suporte teórico a Teoria de Wanda de Aguiar Horta.

\section{Procedimentos éticos}

Pesquisa seguiu as normas propostas pela Resolução $n$ 으 466 de 2012 do Conselho Nacional de Saúde, aprovada pelo Comitê de Ética em Pesquisa local pelo parecer no 2.630.923.

\section{RESULTADOS}

Foram elencados $19 \mathrm{DE}$, com intervenções e resultados esperados, com base na CIPE®, e na teoria de Wanda de Aguiar Horta, apresentados no quadro 1.

Quadro 1: Enunciados de DE, IE e RE elaborados a partir da CIPE®.

$\begin{array}{ll}\begin{array}{ll}\text { Diagnóstico } & \text { Intervenções de Enfermagem }\end{array} & \begin{array}{l}\text { Resultado } \\ \text { esperado }\end{array}\end{array}$

NECESSIDADE PSICOBIOLÓGICA - Integridade Física e Cutaneomucosa

\begin{tabular}{cll}
\hline Alergia & - Evitar coçar o local & Alergia \\
& - Estimular o aleitamento materno & melhorada \\
& - Identificar agentes causadores & \\
& - Utilizar sabonete neutro & \\
& - Encaminhar para o médico pediatra & \\
& - Orientar sinais de alerta e ir ao & \\
& Hospital ou Pronto Atendimento, se & \\
& piora & \\
Alergia a picada & - Usar mosquiteiro ou telas nas & Alergia \\
de inseto & janelas & melhorada \\
& - Manter unhas curtas e limpas & \\
& - Retornar à UBS, caso haja piora do & \\
& quadro clínico ou dúvidas &
\end{tabular}

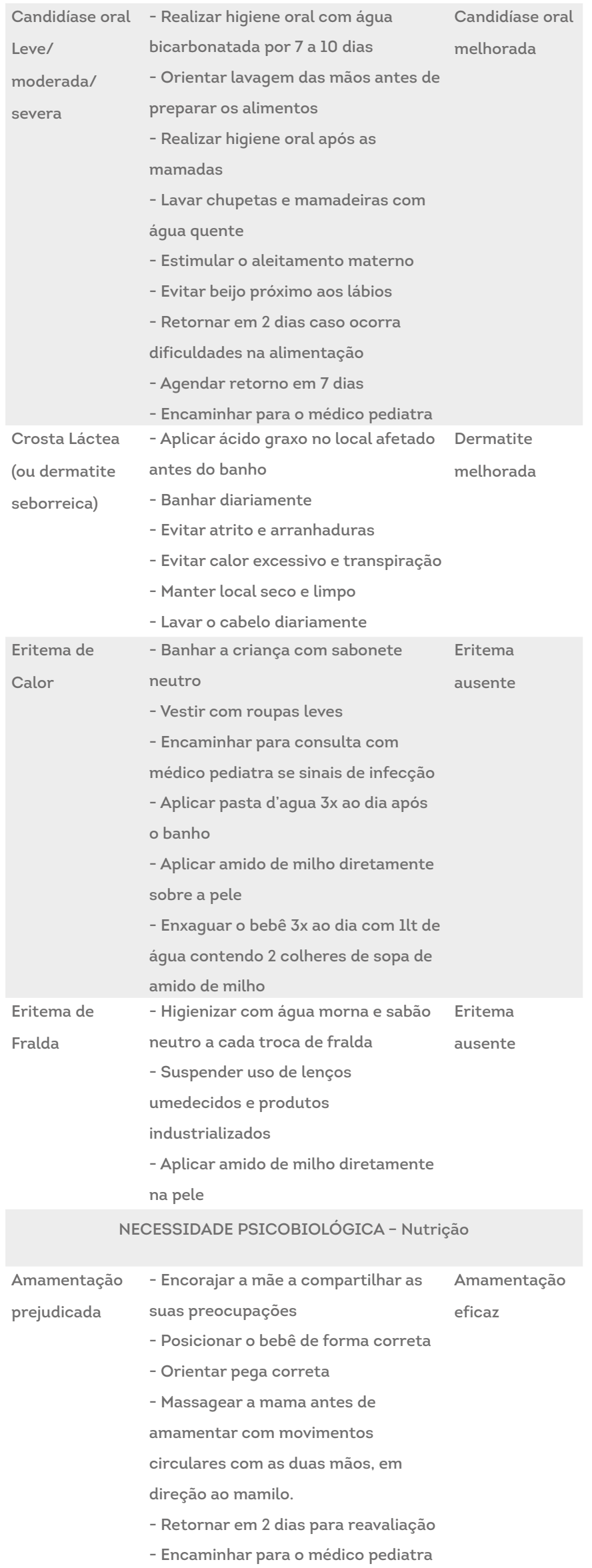




\begin{tabular}{|c|c|c|}
\hline \multirow{2}{*}{$\begin{array}{l}\text { Crescimento } \\
\text { prejudicado }\end{array}$} & - Supervisionar curva de crescimento & \multirow{2}{*}{$\begin{array}{l}\text { Crescimento } \\
\text { adequado }\end{array}$} \\
\hline & $\begin{array}{l}\text { - Encaminhar para avaliação com o } \\
\text { médico pediatra } \\
\text { - Observar biotipo dos pais } \\
\text { - Orientar sobre a importância das } \\
\text { consultas de Puericultura } \\
\text { - Solicitar o acompanhamento por } \\
\text { meio de visita do Agente Comunitário } \\
\text { de Saúde (ACS) }\end{array}$ & \\
\hline $\begin{array}{l}\text { Peso } \\
\text { prejudicado }\end{array}$ & $\begin{array}{l}\text { - Orientar ingesta alimentar } \\
\text { adequada para idade } \\
\text { - Reforçar aleitamento materno } \\
\text { - Encaminhar ao médico pediatra } \\
\text { - Retornar em } 15 \text { dias } \\
\text { - Solicitar avaliação da nutricionista } \\
\text { - Encaminhar para o CRAS/CREAS } \\
\text { - Solicitar o acompanhamento por } \\
\text { meio de visita do ACS }\end{array}$ & Peso Adequado \\
\hline NECESSIDA & $\begin{array}{c}\text { DE PSICOBIOLÓGICA - Percepção Visu } \\
\text { Gustativa, Olfativa, Tátil e Dolorosa }\end{array}$ & lal, Auditiva, \\
\hline $\begin{array}{l}\text { Cólica } \\
\text { abdominal } \\
\text { leve/moderada/ } \\
\text { severa }\end{array}$ & $\begin{array}{l}\text { - Posicionar a criança em decúbito } \\
\text { dorsal e massagear a região } \\
\text { abdominal no sentido horário } \\
\text { - Posicionar a criança em decúbito } \\
\text { dorsal e movimentar as pernas em } \\
\text { direção ao corpo } \\
\text { - Deixar a criança no colo para sentir- } \\
\text { se acolhido e aquecido } \\
\text { - Conferir postura e pega na } \\
\text { amamentação } \\
\text { - Banhar a criança em água morna } \\
\text { para promover o relaxamento } \\
\text { - Reduzir estímulos sonoros e } \\
\text { luminosos }\end{array}$ & $\begin{array}{l}\text { Cólica } \\
\text { abdominal } \\
\text { melhorada }\end{array}$ \\
\hline $\mathrm{NECl}$ & ESSIDADE PSICOBIOLÓGICA - Elimina & ção \\
\hline $\begin{array}{l}\text { Constipação } \\
\text { leve/moderada/ } \\
\text { severa }\end{array}$ & $\begin{array}{l}\text { - Identificar padrão alimentar } \\
\text { - Orientar alimentos ricos em fibras } \\
\text { - Incentivar ingesta hídrica } \\
\text { - Analisar constipação e processo } \\
\text { fisiológico } \\
\text { - Encaminhar para o médico pediatra }\end{array}$ & $\begin{array}{l}\text { Constipação } \\
\text { melhorada }\end{array}$ \\
\hline $\begin{array}{l}\text { Diarreia } \\
\text { Leve/ } \\
\text { moderada/ } \\
\text { severa }\end{array}$ & $\begin{array}{l}\text { - Suspender uso do sulfato ferroso } \\
\text { - Aumentar ingesta hidrica } \\
\text { - Orientar alimentação adequada } \\
\text { - Manter a alimentação habitual, } \\
\text { principalmente o leite materno, } \\
\text { aumentando a frequência das } \\
\text { mamadas } \\
\text { - Oferecer à criança a solução } \\
\text { reidratante; } \\
\text { - Trocar fralda a cada evacuação ou } \\
\text { após urinar para evitar assaduras; } \\
\text { - Encaminhar para o médico pediatra } \\
\text { - Orientar sinais de alerta e ir ao } \\
\text { Hospital ao Pronto Atendimento, } \\
\text { se piora }\end{array}$ & $\begin{array}{l}\text { Diarreia } \\
\text { melhorada }\end{array}$ \\
\hline
\end{tabular}

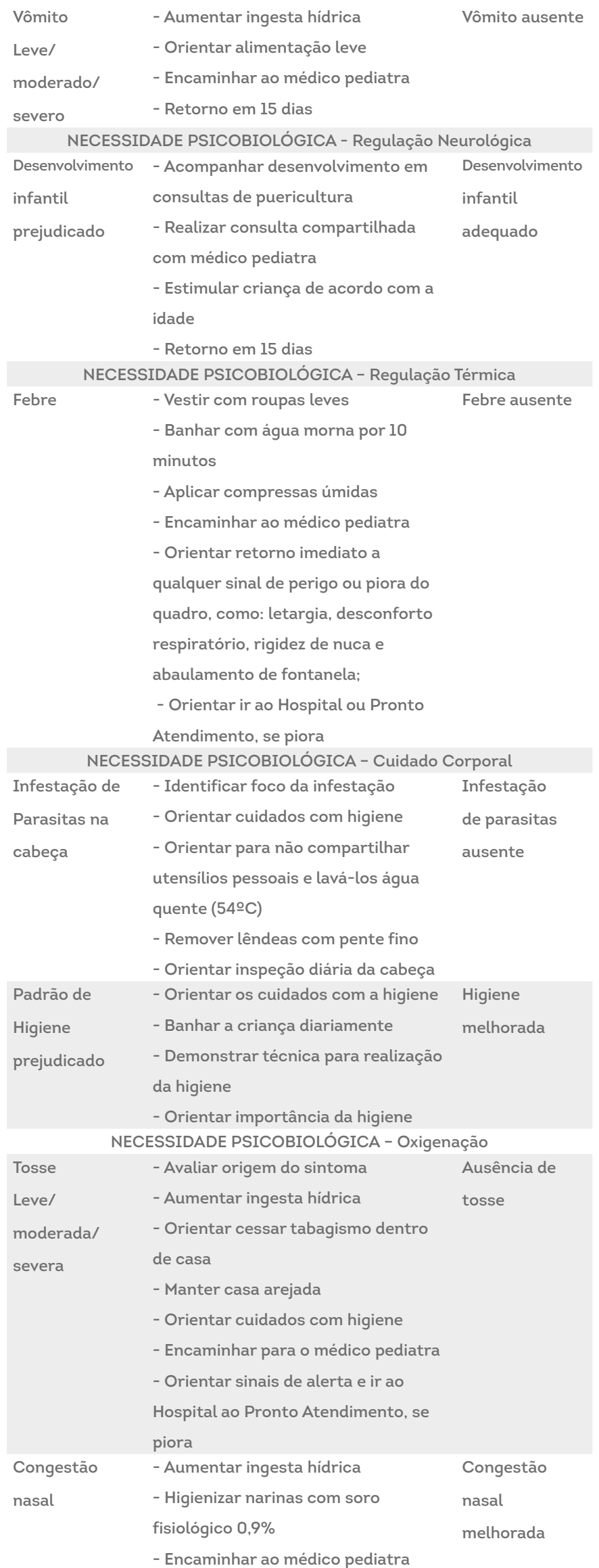




\section{DISCUSSÃO}

$\mathrm{Na}$ infância a criança desenvolve grande parte das suas potencialidades e para acompanhar o ritmo de crescimento e desenvolvimento o enfermeiro necessita ter um olhar global das situações que permeiam a assistência, assim como desenvolver raciocínio clínico para compreender as diferentes situações ${ }^{(10)}$.

A puericultura tem como foco a integralidade do cuidado da criança; é uma atividade dinâmica de baixa complexidade, que possibilita a realização de ações de proteção à saúde e prevenção de agravos ${ }^{(10,11,12,13)}$. Com base nessa afirmativa considera-se importante a construção coletiva de DE, IE e RE a partir de cada realidade, pois eles irão nortear e qualificar as ações do enfermeiro na APS por serem uma linguagem universal entre os profissionais, contribuindo para o processo de assistência à criança ${ }^{(10)}$.

Em relação aos $\mathrm{DE}$, foram elencados os de maior relevância e frequência na rotina de atendimento das crianças, considerando a fase de crescimento e desenvolvimento. Foram predominantes os diagnósticos voltados às necessidades psicobiológicas. Contudo, chama a atenção que os aspectos relacionados às necessidades psicossociais e psicoespirituais, não foram considerados prioritários, contrariando dessa forma a concepção de Horta ${ }^{(14)}$ que entende o ser humano como um ser indivisivel, que comporta todos os aspectos de forma a se interrelacionarem. Ainda, Horta afirma que a Enfermagem é prestada ao ser humano, não aos seus desequilibrios ou doenças, tendo como finalidade promover os processos de promoção da saúde, prevenção e reabilitação de agravos e incentivo ao autocuidado.

Assim, cada criança deverá ser assistida de forma integral no momento da CE, considerando suas necessidades de forma individual e com objetivo de manter um crescimento e desenvolvimento adequados, principalmente nos primeiros anos de vida ${ }^{(15)}$.

Dentre os DE construídos e, olhando para os aspectos biopsicossociais, o diagnóstico de higiene prejudicada chama a atenção porque, além dele, outros aspectos relacionados à higiene apareceram de forma predominante, como as infestações por parasitas, prevenção dos eritemas de calor e de fralda, formas de diminuir os impactos da diarreia. É importante ao se perceber que as condições de higiene têm reflexos negativos, orientar a família sobre os cuidados com o corpo e de como eles reduzem problemas comuns, como os relacionados a obstruções nasais, desconfortos comuns e indisposições durante as mamadas ${ }^{(16)}$.

Estudo $^{(17)}$ que analisou 400 prontuários de crianças de zero a 36 meses, atendidas em CE, também identificou a prevalência de problemas relacionados a aspectos cutaneosmucosos, mesmo em consultas de rotina, demonstrando a importância de diagnósticos que possibilitem a identificação e intervenção precoce desses achados clínicos.
Outrodiagnósticoimportanteéorelacionadoaamamentação, que além de contemplar aspectos sociais, culturais, históricos, emocionais tem foco na criança, mãe e família. No DE amamentação ineficaz, além das condições biológicas, deve ser avaliada questões relacionadas ao conhecimento, capacidade e crenças da mulher sobre a amamentação que podem influenciar de maneira negativa esse processo. As orientações referentes ao aleitamento materno são primordiais, e devem fazer parte da CE, pois ele possui efeito protetor para alergias, infecções, diarreia, manutenção do crescimento adequado(10). A avaliação do crescimento da criança está atrelada a dois DE importantes - peso e crescimento prejudicados. Além da avaliação do peso, altura e perímetro cefálico, é importante que o enfermeiro tenha uma visão global do crescimento, observando fatores influenciadores as condições de vida, alimentação e fase em que a criança está no momento da avaliação(18).

Na avaliação do crescimento, o peso deve ser observado com atenção pelo enfermeiro, principalmente, por ser uma medida que sofre variações e indica aspectos biológicos e sociais. Contudo, uma medida pouco referida nos estudos é o Índice de Massa Corporal (IMC), porém de relevância, pois permite avaliar de maneira global a criança ao cruzar as variáveis peso, estatura e idade. A pouca atenção ao IMC pode estar relacionada a sua inserção recente na Caderneta de Saúde da Criança como critério para o acompanhamento infantil(18).

Observou-se, ainda, a ausência de DE relacionados a promoção da saúde, provavelmente, devido ao cotidiano dessas profissionais estar voltado para ações de prevenção ou reabilitação de agravos. Os 19 DE elencados refletem visão focada nos processos biológicos, com ênfase em agravos já estabelecidos. Quando a assistência é voltada para o curativismo ela fragmenta o cuidado, geralmente, valorizando dimensões biológicas e o adoecer, indo contra as diretrizes da APS, que têm como base a proteção e promoção em saúde ${ }^{(12,19)}$

Um dos fatores que contribui para a escolha de DE voltados para aspectos de doença é o atendimento em livre demanda; nessas ocasiões, a procura pela consulta, comumente, é relacionada a queixas agudas. Ou seja, existe mais busca pelo atendimento imediato, com foco agudo, do que pela CE programada, em que o objetivo é estabelecer um cuidado integral com vistas à promoção da saúde e prevenção de agravos. A livre demanda pode estar associada à ampliação do acesso às UBS, fazendo com o que o usuário procure atendimento mais rápido e facilitado, incidindo diretamente na consulta programada ${ }^{(20)}$.

Nesse sentido, reforça-se a importância de o enfermeiro buscar ferramentas de trabalho que tornam a CE resolutiva, como por exemplo, a construção de DE, IE e RE com base na CIPE que vai ao encontro da necessidade de padronizar e qualificar a assistência de enfermagem em puericultura, garantindo respaldo científico. 


\section{Limitações do estudo}

As limitações deram-se pela escassez de artigos técnicos para embasar os argumentos utilizados na discussão. Foi um desafio discutir de forma técnica e cientifica os achados referentes aos DE, IE e RE elencados com base na prática das enfermeiras da APS.

\section{Contribuições do Estudo}

Esse estudo contribuiu para a qualificação profissional das enfermeiras da APS e, consequentemente, no aumento da resolutividade nas CE à criança. Além disso serviu como base para a revisão do Protocolo Municipal de Saúde da Criança do municipio onde o estudo foi realizado.

\section{CONSIDERAÇÕES FINAIS}

A escolha dos DE, IE e RE realizada coletivamente, favorece a assistência qualificada e padronizada. Contudo, é imprescindível que o enfermeiro aprimore seu conhecimento técnico e cientifico para determinar quais intervenções são necessárias no momento da CE, além de que a revisão, inclusão e exclusão de DE, IE e RE deve ser contínua e permanente, visando torná-los exequíveis. É importante estimular o enfermeiro para a consolidação da sua autonomia uma vez que ela melhora a qualidade do cuidado. Essa pesquisa pode subsidiar os enfermeiros para executarem ações assertivas, auxiliando-os na CE ao apresentar alguns DE, IE e RE que orientam o atendimento à criança, proporcionando segurança, qualificando e otimizando a assistência prestada. Contudo, é necessário que se dê continuidade na construção de outros DE, IE e RE, assim como no aperfeiçoamento dos já elencados.

\section{Contribuições dos autores}

Concepção e/ou desenho: Ingrid Pujol Hanzen, Elisangela Argenta Zanatta; análise e interpretação dos dados, redação do artigo, revisão crítica, revisão final: Ingrid Pujol Hanzen, Elisangela Argenta Zanatta, Silvana dos Santos Zanotelli.

Agradecimentos e financiamento: À UDESC e a CAPES/COFEN que financiaram essa pesquisa pelo Edital $n$ 으 27/2016.

\section{REFERÊNCIAS}

1. Soares DG, Pinheiro MCX, Queiroz DM de, Soares DG. Implantação da puericultura e desafios do cuidado na estratégia saúde da familia em um municipio do Estado do Ceará. Revista Brasileira em Promoção da Saúde. [Internet]. 2016: 29(1):132-138. Available From: http://periodicos.unifor.br/RBPS/article/ view/3713.

2. Ministério da Saúde (BR). Política Nacional de Atenção à Saúde da Criança: orientações para implementação. 2018. Available from: http://www.saude.pr. gov.br/arquivos/File/Politica_Nacional_de_Atencao_Integral_a_Saude_da_ Crianca_PNAISC. pdf

3. Gubert FA, Santos DAS, Pinheiro MTM, Brito LLMS, Pinheiro SRCS, Martins MC. Protocolo de Enfermagem para consulta de puericultura. Revista da Rede de Enfermagem do Nordeste. [Internet]. 2015: 16 (1):81-9. Available From: http://www.redalyc.org/pdf/3240/324036185010.pdf

4. Conselho Federal de Enfermagem. Resolução COFEN no 358 de 2009. Sistematização da Assistência de Enfermagem e a implementação do Processo de Enfermagem em ambientes públicos ou privados, em que ocorra o cuidado profissional de Enfermagem. 2009. Available From: http://www.cofen.gov.br/ resoluo-cofen-3582009_4384.html

5. Primo CC, Resende FZi, Garcia TR, Duran ECM, Brandão MAG. Subconjunto terminológico da CIPE ${ }^{8}$ para assistência à mulher e à criança em processo de amamentação. Rev. Gaúcha Enferm. [Internet]. 2018: 39. e2017-0010. Available From: http://www.scielo.br/scielo.php?script=sci_arttextEpid=S1983-14472018000100410\&lng=pt.

6-. Garcia TR, Bartz CC. Coenen AM. CIPE: uma linguagem padronizada para a prática profissional. In: Garcia TR (Org.). Classificação Internacional para a prática de enfermagem CIPE®. Porto Alegre: Artmed, 2018.

7. Thiollent M. Metodologia da pesquisa-ação. 18. ed. São Paulo: Cortez, 2011.

8. Kitzinger, J. Grupos Focais. In: POPE, C.: MAYS, N. (Org.). Pesquisa qualitativa na atenção à saúde. 3. ed. Porto Alegre: Artmed, 2009

9. Polit-O'hara D, Beck CT. Fundamentos de pesquisa em enfermagem: avaliação de evidências para prática da enfermagem. 7. ed. Porto Alegre: Artes Médicas, 2011

10. Dantas AMN, Gomes GLL, Silva K de L, Nóbrega MML da.

Diagnósticos de enfermagem para as etapas do crescimento e desenvolvimento de crianças utilizando a CIPE@. Revista Eletrônica de Enfermagem. [Internet]. 2016; 18:ell. Available From: https://revistas.ufg.br/fen/article/ view/35524/21457

11. Monteiro FPM, Araujo TL, Costa FBC, Leandro TA, Cavalcante TF, Lopes MVO. Validação clínica do diagnóstico de enfermagem disposição para desenvolvimento melhorado do lactente. Rev Bras Enferm [Internet]. 2016:69(5):802-
Available From: http://www.scielo.br/pdf/reben/v69n5/0034-7167-reben-69-05-0855.pdf

12. Vieira DS, Santos NCCB, Nascimento JA do, Collet N, Toso BRGO, Reichert APS. A prática do enfermeiro na consulta de puericultura na Estratégia Saúde da Familia. Texto contexto - enferm. [Internet]. 2018: 27( 4 ): e4890017. Available Fron: http://www.scielo.br/pdf/tce/v27n4/0104-0707-tce-27-04-e4890017.pdf

13. Santos GS, Pieszak GM, Gomes GC, Biazus CB, Silva SO. Contribuições da Primeira Infância Melhor para o crescimento e desenvolvimento infantil na percepção das familias. Rev Fun Care Online. [Internet]. 2019: 11(1):67-73. Available From: http://www.seer.unirio.br/index.php/cuidadofundamental/article/ view/646

14. Horta, WA. Processo de Enfermagem. São Paulo: EPU, 1979

15. Costa P, Duarte AP, Belela-Anacleto ASC, Andrade PR, Balieiro MMFG Veríssimo MDLOR. Diagnósticos de enfermagem em consultas de atenção primária à saúde de recém-nascidos. Rev.Bras.Enferm. [internet], v. 71, n.6, p. 2961-8, 2018. DOI: http://dx.doi.org/10.1590/0034-7167-2017-0954 Disponive em:<http://www.scielo.br/pdf/reben/v7ln6/pt_0034-7167-reben-71-06-2961. pdf>. Acesso em: 04 abr. 2019

16. Santos KH dos, Marques D. Diagnósticos de Enfermagem na Atenção Básica: contributos da Classificação Internacional para a Prática de Enfermagem. Revista da Sociedade Brasileira de Enfermeiros Pediatras. [Internet]. 2015 15(2):108-113. Available From: https://sobep.org.br/revista/component/zine/ article/214-diagnosticos-de-enfermagem-na-atencao-basica-contributos-da-classificacao-internacional-para-a-pratica-de-enfermagem.html

17. Monteiro ATA, Ferrari RAP, Tacla MTGM , Souza ALDM de. Consulta de enfermagem à criança após alta das maternidades: seguimento na atenção primária. Rev. Soc. Bras. Enferm. Ped. [Internet]. 2017; 17(1): 7-13. Available From: https://sobep.org.br/revista/images/stories/pdf-revista/voll7-nl/vol_17_n_ 1-artigo_original_l.pdf

18. Gaiva MAM Monteschio CAC Moreira MDS, Salge AKM Avaliação do crescimento e desenvolvimento infantil na consulta de enfermagem. Av Enferm. [Internet].2018;36(1)9-21. Available From: http://www.scielo.org.co/pdf/aven/ v36nl/0121-4500-aven-36-01-00009.pdf.

19. Malaquias TSM, Baldissera VAD, Higarashi IH. Percepções da equipe de saúde e de familiares sobre a consulta de puericultura. Rev. Cogitare Enfermagem [Internet]. 2015; 20(2):367-373. Available from: http://www.redalyc.org/articulo.oa?id=483647679017.

20. Kahl C, Meirelles BHS, Lanzoni GMM, Koerich C, Cunha KS. Actions and interactions in clinical nursing practice in Primary Health Care. Rev Esc Enferm USP. 2018;52:e03327. Available From: http://dx.doi.org/10.1590/S1980$220 \times 2017025503327$ 